\title{
Functional photoacoustic tomography for non-invasive imaging of cerebral blood oxygenation and blood volume in rat brain in vivo
}

Xueding Wang, Xueyi Xie, Geng Ku, George Stoica, Lihong V. Wang

Xueding Wang, Xueyi Xie, Geng Ku, George Stoica, Lihong V. Wang, "Functional photoacoustic tomography for non-invasive imaging of cerebral blood oxygenation and blood volume in rat brain in vivo," Proc. SPIE 5697, Photons Plus Ultrasound: Imaging and Sensing 2005: The Sixth Conference on Biomedical Thermoacoustics, Optoacoustics, and Acousto-optics, (25 April 2005); doi: 10.1117/12.589680

SPIE. Event: SPIE BiOS, 2005, San Jose, CA, United States 


\title{
Functional photoacoustic tomography for non-invasive imaging of cerebral blood oxygenation and blood volume in rat brain in vivo
}

\author{
Xueding Wang ${ }^{\mathrm{a}}$, Xueyi Xie ${ }^{\mathrm{a}}$, Geng Ku ${ }^{\mathrm{a}}$, George Stoica ${ }^{\mathrm{b}}$, and Lihong V. Wang ${ }^{* a}$ \\ ${ }^{a}$ Optical Imaging Laboratory, Department of Biomedical Engineering, Texas A\&M University, \\ 3120 TAMU, College Station, Texas 77843-3120 \\ ${ }^{b}$ Department of Pathobiology, Texas A\&M University, College Station, TX 77843-5547
}

\begin{abstract}
Based on the multi-wavelength laser-based photoacoustic tomography, non-invasive in vivo imaging of functional parameters, including the hemoglobin oxygen saturation and the total concentration of hemoglobin, in small-animal brains was realized. The high sensitivity of this technique is based on the spectroscopic differences between oxy- and deoxy-hemoglobin while its spatial resolution is bandwidth-limited by the photoacoustic signals rather than by the optical diffusion as in optical imaging. The point-by-point distributions of blood oxygenation and blood volume in the cerebral cortical venous vessels, altered by systemic physiological modulations including hyperoxia, normoxia and hypoxia, were visualized successfully through the intact skin and skull. This technique, with its prominent intrinsic advantages, can potentially accelerate the progress in neuroscience and provide important new insights into cerebrovascular physiology and brain function that are of great significance to the neuroscience community.
\end{abstract}

Keywords: photoacoustic tomography, functional imaging, spectroscopic imaging, animal brain, blood oxygenation, blood volume, optical contrast, high resolution.

\section{INTRODUCTION}

Photoacoustic tomography (PAT, also referred to as optoacoustic or thermoacoustic tomography) ${ }^{1-7}$ involves both light and ultrasound. In PAT, a short-pulsed electromagnetic source - a laser or an RF source - is used to irradiate a biological tissue sample under investigation. Due to the absorption of the electromagnetic energy by the absorbing chromophores in the sample (e.g. hemoglobin and melanosome), a spatially distributed temperature rise of the order of $\mathrm{mK}$ will be produced in a short time frame. Consequently, the thermoelastic expansion of tissues will cause the emission of ultrasonic waves, referred to as photoacoustic waves. The electromagnetic heating must be rapid (on the order of nanoseconds) in order to produce photoacoustic waves efficiently; in other words, static temperature distribution or slow heating does not produce photoacoustic waves. The photoacoustic waves are measured by a wideband ultrasonic transducer(s) scanning around the sample. The acquired photoacoustic waves are used to reconstruct the distribution of optical absorption in the sample. Because the laser pulse is short, proportionately high frequency ultrasonic waves will be produced and used to provide diffraction-limited spatial resolution.

The study of functional changes in regional blood oxygenation (hemoglobin oxygen saturation) and blood volume (total concentration of hemoglobin) is important to diagnostic imaging and therapeutic monitoring. Despite years of effort, however, no imaging modality has been developed previously that is clinically feasible for the accurate, continuous and non-invasive monitoring of hemoglobin oxygen saturation in distant organs, such as brain. The brain is highly responsive to hemodynamic changes including hemoglobin oxygen saturation. Rapid high-resolution mapping of functional parameters in the brain, e.g. cerebral blood oxygenation and blood volume, is of great significance in neurophysiology, neuropathology, and neurotherapy, for example in visualizing the functional parameters of brain cancers and traumatic brain injuries, monitoring ischemia and shock, and studying neural activities. Near-infrared spectroscopy (NIRS), based on the spectroscopic differences between oxy- and deoxy-hemoglobin, has long been

*Corresponding author: email: Lwang@tamu.edu; phone: 1979 847-9040; fax: 1979 845-4450 
explored for the non-invasive measurement of blood oxygenation and blood flow. ${ }^{8-10}$ However, because of the overwhelming scattering of light in biological tissues, this technique has so far proved incapable of providing satisfactory spatial resolution and accuracy for imaging deep tissues beyond the skin.

PAT combines the high contrast advantage of optical imaging and the high resolution advantage of ultrasonic imaging. The contrast in PAT is determined primarily by the optical properties of biological tissues, and the spatial resolution in PAT is determined primarily by the photoacoustic waves originating from within biological tissues rather than by optical diffusion as in optical imaging. ${ }^{11}$ Having achieved non-invasive photoacoustic imaging of small-animal brains based on the intrinsic optical contrast among various brain tissues, ${ }^{5,6}$ we turned our attention to the in vivo functional photoacoustic imaging of cerebral blood oxygenation and blood volume in small-animal brains based on the spectroscopic differences between oxy- and deoxy-hemoglobin.

\section{METHODS}

The experimental system for functional photoacoustic imaging of blood oxygenation and blood volume in rat brain cerebral cortex is shown in Fig. 1. A tunable dye laser (ND6000, Continuum) pumped by an Nd:YAG laser (Brilliant B, Bigsky) provided irradiation pulses. The laser pulse duration was $6.5 \mathrm{~ns}$ at the full width at half maximum (FWHM). The pulse repetition rate was $10 \mathrm{~Hz}$. The laser beam was expanded and homogenized by a concave lens and, subsequently, by a piece of ground glass before it reached the rat head. The incident energy density of the laser beam on the surface of the rat head was controlled to $<3 \mathrm{~mJ} / \mathrm{cm}^{2}$ (much lower than the ANSI safety limit ${ }^{12}$ ), which, attenuated and homogenized further by the skin and skull, induced a temperature rise in the brain vessels estimated to be $<15 \mathrm{mK}$. An unfocused ultrasonic transducer (XMS-310, Panametrics) was driven by a computer-controlled step motor to scan circularly around the rat brain cortex in the horizontal plane with a scanning radius of $\sim 3 \mathrm{~cm}$ and a scanning step size of $1.5^{\circ}$. The transducer had a central frequency of $10.4 \mathrm{MHz}$, a bandwidth of $\sim 100 \%$ at $-6 \mathrm{~dB}$, and a diameter of active element of $2 \mathrm{~mm}$. The photoacoustic signals received by the transducer were sent to an oscilloscope (TDS 540B, Tektronics) through an amplifier. Finally, the digitized photoacoustic signals were collected by the computer for image reconstruction employing a modified back-projection algorithm. ${ }^{11}$

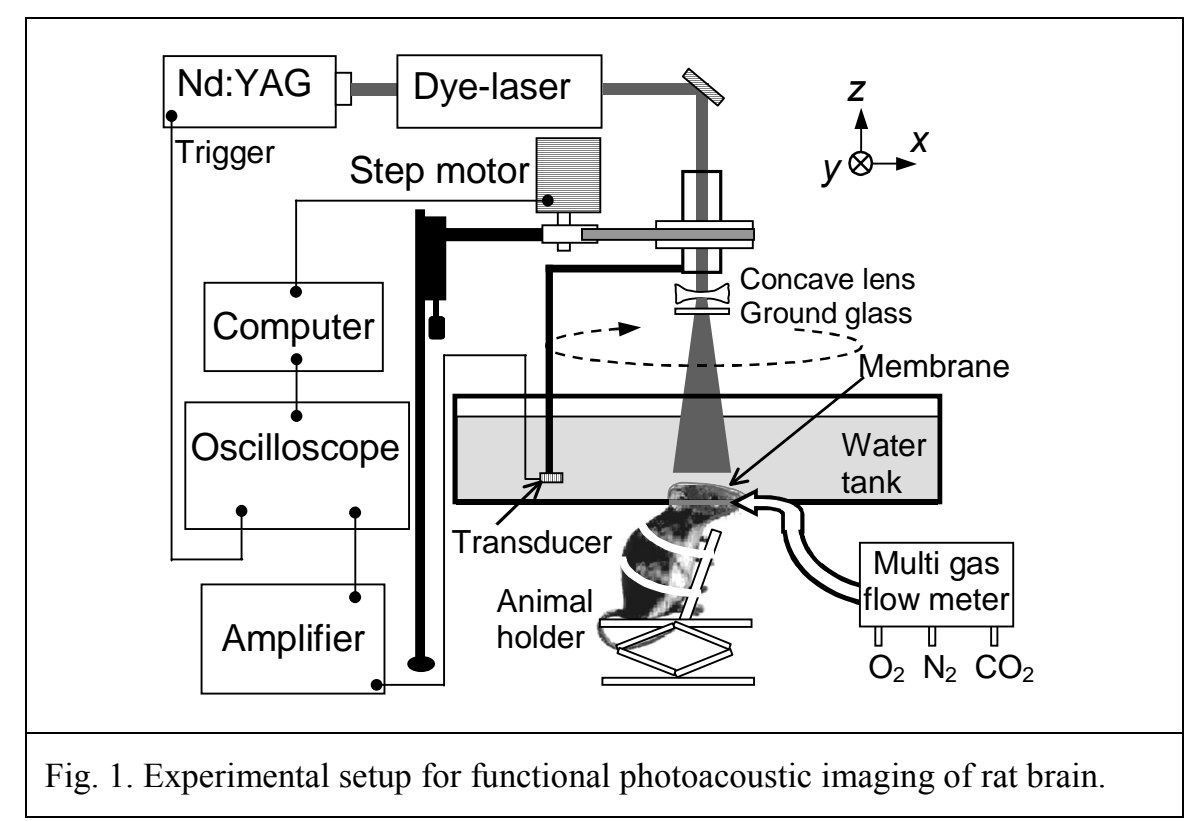

We performed functional photoacoustic imaging of rat brains in vivo to evaluate the capability of this technique for simultaneously assessing the cerebral blood volume and the oxygenation level of hemoglobin. Sprague Dawley rats ( $\sim 80 \mathrm{~g}$, Charles River Breeding Laboratories) were employed in this study. Before imaging, the hair on the head of the 
rat was removed using hair remover lotion. A dose of $87 \mathrm{mg} / \mathrm{kg}$ Ketamine plus Xylasine $13 \mathrm{mg} / \mathrm{kg}$, administered intramuscularly, was used to anesthetize the rat and supplemental injections of a similar anesthetic mixture $(\sim 50$ $\mathrm{mg} / \mathrm{kg}$ /hour) kept the rat motionless throughout the experiment. The rat head, fixed steadily by a homemade animal holder and a breathing mask, protruded up into a water tank through a hole in the bottom of the tank. A piece of clear membrane between the water and the rat head sealed the hole. Through the breathing mask, inhaled gas was ventilated to the rat at a flow rate of $\sim 0.6 \mathrm{l} / \mathrm{min}$. The oxygen concentration in the inhaled gas was adjustable through a gas proportioner meter (GMR2, Aalborg). During the experiment, the sensor of a Pulse Oximeter (8600 V, Nonin Medical, Inc.) clamped a hind foot of the rat to monitor the global arterial blood oxygenation. Through the modulation of the inhaled oxygen concentration, the rat experienced three systemic physiological statuses-hyperoxia, normoxia and hypoxia. The observed arterial blood oxygenation levels of the rat under these three physiological statuses were $99 \pm 1 \%$, $92 \pm 2 \%$, and $81 \pm 4 \%$ respectively according to the Pulse Oximeter. Under each status, photoacoustic imaging of the rat brain cerebral cortex was implemented with laser light at two wavelengths respectively. After photoacoustic images were taken, the rat was sacrificed immediately using pentobarbital $(120 \mathrm{mg} / \mathrm{kg}$, IP). The skin and skull of the rat were removed and an open-skull photograph of the brain cortex was taken as a control.

Similar to NIRS, photoacoustic measurement of blood oxygen saturation and blood volume relies on the spectroscopic differences between oxy-hemoglobin $\left(\mathrm{HbO}_{2}\right)$ and deoxy-hemoglobin $(\mathrm{Hb}) \cdot{ }^{13-16}$ When $\mathrm{HbO}_{2}$ and $\mathrm{Hb}$ are the dominant absorbing chromophores in a biological sample (which was the case in this study), the concentrations of these two forms of hemoglobin can be computed using the measured optical absorptions of the sample at two wavelengths. For instance, the absorption coefficients of a blood sample at two wavelengths, $\lambda_{1}$ and $\lambda_{2}$, can be expressed as

$$
\begin{aligned}
& \mu_{a}^{\lambda_{1}}=\varepsilon_{\mathrm{Hb}}^{\lambda_{1}}[\mathrm{Hb}]+\varepsilon_{\mathrm{HbO}_{2}}^{\lambda_{1}}\left[\mathrm{HbO}_{2}\right], \\
& \mu_{a}^{\lambda_{2}}=\varepsilon_{\mathrm{Hb}}^{\lambda_{2}}[\mathrm{Hb}]+\varepsilon_{\mathrm{HbO}_{2}}^{\lambda_{2}}\left[\mathrm{HbO}_{2}\right],
\end{aligned}
$$

where $\mu_{a}\left(\mathrm{~cm}^{-1}\right)$ is the absorption coefficient; $\varepsilon_{\mathrm{Hb}}$ and $\varepsilon_{\mathrm{HbO} 2}$ are the known molar extinction coefficients $\left(\mathrm{cm}^{-1} \mathrm{M}^{-1}\right)$ of the oxy- and deoxy-hemoglobin, respectively; and $[\mathrm{Hb}]$ and $\left[\mathrm{HbO}_{2}\right]$ are, respectively, the concentrations (M) of the two forms of hemoglobin. With the measured absorption coefficients of the blood sample at the two wavelengths, the concentrations of $\mathrm{HbO}_{2}$ and $\mathrm{Hb}$ can be computed by solving the following equations:

$$
\begin{gathered}
{\left[\mathrm{HbO}_{2}\right]=\frac{\varepsilon_{\mathrm{Hb}}^{\lambda_{2}} \mu_{a}^{\lambda_{1}}-\varepsilon_{\mathrm{Hb}}^{\lambda_{1}} \mu_{a}^{\lambda_{2}}}{\varepsilon_{\mathrm{Hb}}^{\lambda_{2}} \varepsilon_{\mathrm{HbO}}^{\lambda_{1}}-\varepsilon_{\mathrm{Hb}}^{\lambda_{1}} \varepsilon_{\mathrm{HbO}_{2}}^{\lambda_{2}}},} \\
{[\mathrm{Hb}]=\frac{\varepsilon_{\mathrm{HbO}_{2}}^{\lambda_{1}} \mu_{a}^{\lambda_{2}}-\varepsilon_{\mathrm{HbO}_{2}}^{\lambda_{2}} \mu_{a}^{\lambda_{1}}}{\varepsilon_{\mathrm{Hb}}^{\lambda_{2}} \varepsilon_{\mathrm{HbO}_{2}}^{\lambda_{1}}-\varepsilon_{\mathrm{Hb}}^{\lambda_{1}} \varepsilon_{\mathrm{HbO}_{2}}^{\lambda_{2}}} .}
\end{gathered}
$$

We can further compute functional parameters, including hemoglobin oxygen saturation $\left(\mathrm{SO}_{2}\right)$ and total hemoglobin concentration $(\mathrm{HbT})$, by solving the following two equations:

$$
\begin{aligned}
& \mathrm{HbT}=\left[\mathrm{HbO}_{2}\right]+[\mathrm{Hb}]=\frac{\mu_{a}^{\lambda_{1}} \Delta \varepsilon_{\mathrm{Hb}}^{\lambda_{2}}-\mu_{a}^{\lambda_{2}} \Delta \varepsilon_{\mathrm{Hb}}^{\lambda_{1}}}{\varepsilon_{\mathrm{Hb}}^{\lambda_{1}} \varepsilon_{\mathrm{HbO}_{2}}^{\lambda_{2}}-\varepsilon_{\mathrm{Hb}}^{\lambda_{2}} \varepsilon_{\mathrm{HbO}}^{\lambda_{1}}}, \\
& \mathrm{SO}_{2}=\frac{\left[\mathrm{HbO}_{2}\right]}{\left[\mathrm{HbO}_{2}\right]+[\mathrm{Hb}]}=\frac{\mu_{a}^{\lambda_{2}} \varepsilon_{\mathrm{Hb}}^{\lambda_{1}}-\mu_{a}^{\lambda_{1}} \varepsilon_{\mathrm{Hb}}^{\lambda_{2}}}{\mu_{a}^{\lambda_{1}} \Delta \varepsilon_{\mathrm{Hb}}^{\lambda_{2}}-\mu_{a}^{\lambda_{2}} \Delta \varepsilon_{\mathrm{Hb}}^{\lambda_{1}}},
\end{aligned}
$$

In the experiment of functional PAT of rat brains, the tunable dye laser was used to image the brain cerebral cortex at $584 \mathrm{~nm}$ and $600 \mathrm{~nm}$ respectively. These two images, after they were normalized by considering the incident light energy densities and the attenuations of light energy by the skin and skull, presented the distributions of the relative optical absorptions in the cerebral cortex corresponding to the two wavelengths. With these two images, we estimated point-bypoint functional parameters in the vasculature in the brain cerebral cortex through Eqs. (5) and (6), including $\mathrm{SO}_{2}$ (i.e. blood oxygenation) and $\mathrm{HbT}$ (i.e. blood volume). 


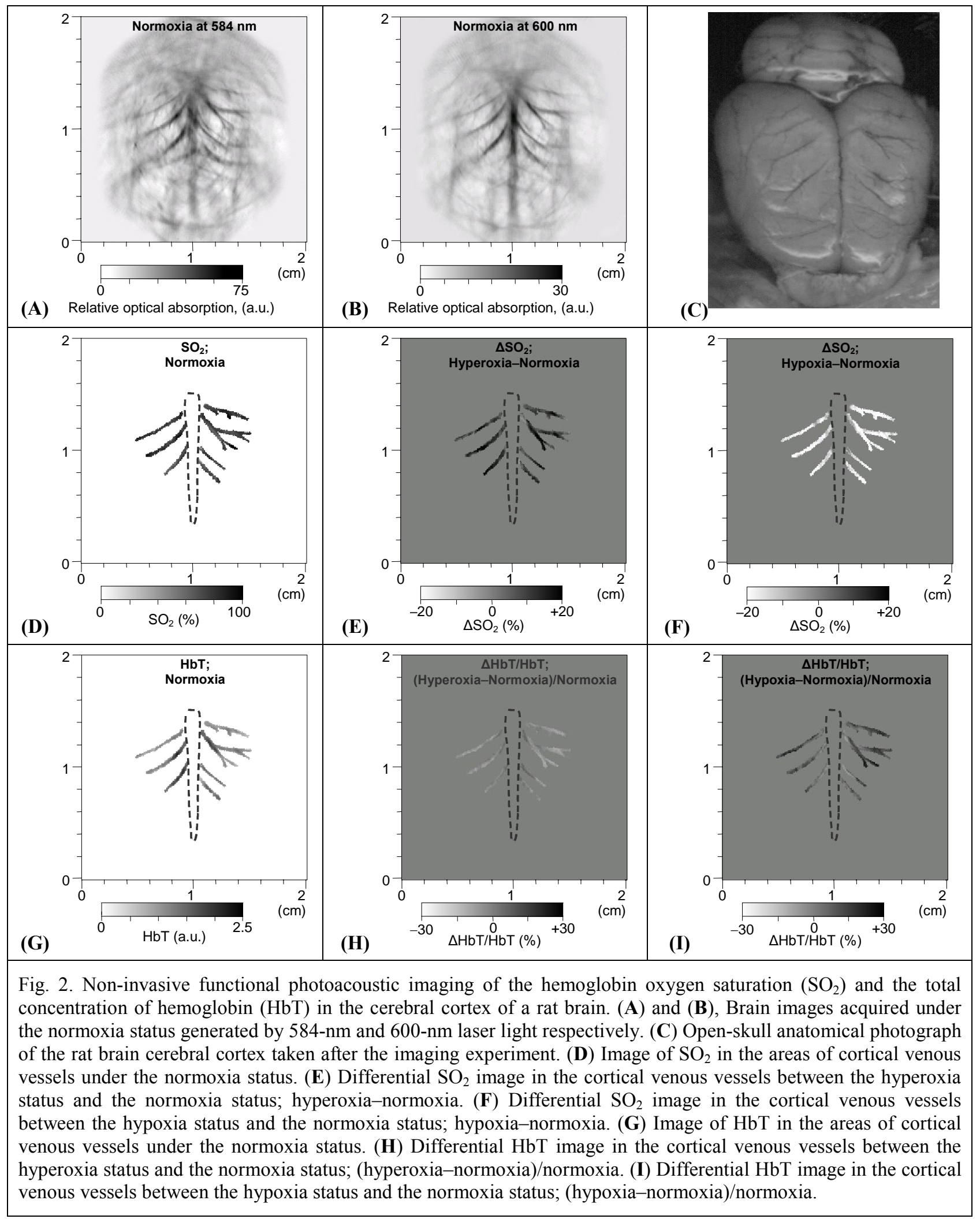




\section{RESULTS}

Figures 2 (A)-(B) are the photoacoustic images of a rat brain acquired under the normoxia status generated by the 584$\mathrm{nm}$ and $600-\mathrm{nm}$ laser light respectively. These two images present the distributions of the relative optical absorptions in the brain cerebral cortex at the two applied wavelengths. The brain images acquired under the hyperoxia and hypoxia statuses show the same vascular structure but different magnitudes of optical absorption. With high absorption contrast between blood and other brain tissues, these structural brain images describe the vascular branches in the cerebral cortex clearly and match well with the open-skull anatomical photograph shown in Fig. 2 (C) that was taken after the imaging experiment.

The areas of the large vascular branches in these structural images, which are all cortical venous vessels, were segmented from the background. Then, with Eqs. (5) and (6), we computed the point-by-point functional parameters, including both $\mathrm{SO}_{2}$ and $\mathrm{HbT}$, in the areas of cortical venous vessels under the three physiological statuses. Fig. 2 (D) shows the image of blood oxygenation in the areas of cerebral cortical venous vessels under the normoxia status. The image in Fig. 2 (E) shows the differential blood oxygenation in the segmented vascular areas between the hyperoxia status and the normoxia status, hyperoxia-normoxia; while the image in Fig. 2 (F) shows the differential blood oxygenation in the segmented vascular areas between the hypoxia status and the normoxia status, hypoxia-normoxia. Fig. $2(\mathrm{G})$ shows the image of blood volume in the areas of cerebral cortical venous vessels under the normoxia status. The image in Fig. $2(\mathrm{H})$ shows the differential blood volume in the segmented vascular areas between the hyperoxia status and the normoxia status, (hyperoxia-normoxia)/normoxia; while the image in Fig. 2 (I) shows the differential blood volume in the segmented vascular areas between the hypoxia status and the normoxia status, (hypoxianormoxia)/normoxia.

When the rat was under the hypoxia status, in the functional photoacoustic images, we observed a decrease in blood oxygenation and an increase in blood volume in the areas of cerebral cortical venous vessels. We also observed an increase in blood oxygenation and a little decrease in blood volume when the rat was under the hyperoxia status. The phenomena observed in this functional spectroscopic PAT experiment agree with the well-established physiology of the brain. ${ }^{17,18}$

\section{CONCLUSION}

For the first time to our knowledge, PAT was employed to image cerebral blood oxygenation and blood volume in small-animal brains in vivo. Photoacoustic technology is able to assess the oxy- and deoxy-hemoglobin simultaneously in a localized area with spatial resolution much better than diffuse optical imaging. Consequently, the point-by-point hemodynamic changes in organs, such as the brain, may be evaluated accurately by using multi-wavelength spectroscopic PAT. This non-ionizing, non-invasive, novel functional imaging technology can potentially provide important new insights into cerebrovascular physiology and brain function that should be of great significance to the neuroscience community.

\section{REFERENCES}

1. C. G. A. Hoelen, F. F. M. de Mul, R. Pongers, and A. Dekker, "Three-dimensional photoacoustic imaging of blood vessels in tissue," Opt. Lett. 23, 648-650 (1998).

2. R. A. Kruger, D. R. Reinecke, and G. A. Kruger, "Thermoacoustic computed tomography-technical considerations," Med. Phys. 26, 1832-1837 (1999).

3. R. O. Esenaliev, A. A. Karabutov, and A. A. Oraevsky, "Sensitivity of laser opto-acoustic imaging in detection of small deeply embedded tumors," IEEE J. Sel. Top. Quant. 5, 981-988 (1999).

4. K. P. Köstli, D. Frauchiger, J. J. Niederhauser, G. Paltauf, H. P. Weber, and M. Frenz, "Optoacoustic imaging using a three-dimensional reconstruction algorithm,” IEEE J. Sel. Top. Quant. 7, 918-923 (2001). 
5. X. Wang, Y. Pang, G. Ku, X. Xie, G. Stoica, and L. V. Wang, "Non-invasive laser-induced photoacoustic tomography for structural and functional imaging of the brain in vivo," Nat. Biotech. 21, $803-806$ (2003).

6. X. Wang, Y. Pang, G. Ku, G. Stoica, and L. V. Wang, "Three-dimensional laser-induced photoacoustic tomography of mouse brain with the skin and skull intact," Opt. Lett. 28, 1739-1741 (2003)

7. G. Ku, X. Wang, G. Stoica, and L. V. Wang, "Multiple-bandwidth photoacoustic tomography", Phys. Med. Biol. 49, 1329-1338 (2004).

8. G. A. Millikan, "The oximeter, an instrument for measuring continuously the oxygen saturation of arterial blood in man,” Rev. Sci. Instrum. 13, 434-444 (1942).

9. F. F. Jöbsis, "Noninvasive, infrared monitoring of cerebral and myocardial oxygen sufficiency and circulatory parameters," Science 198, 1264-1267 (1977).

10. A. Villringer and B. Chance, "Non-invasive optical spectroscopy and imaging of human brain function," Trends. Neurosci. 20, 435-442 (1997).

11.M. Xu and L.-H. Wang, "Time-domain reconstruction for thermoacoustic tomography in a spherical geometry," IEEE T. Med. Imaging 21, 814-822 (2002).

12. American National Standards Institute, American national standard for the safe use of lasers. Standard Z136.1-2000. (ANSI, Inc., New York, NY, 2000)

13.H. Liu, D. A. Boas, Y. Zhang, A. G. Yodh, and B. Chance, "Determination of optical properties and blood oxygenation in tissue using continuous NIR light," Phys. Med. Biol. 40, 1983-1993 (1995).

14. R. L. Barbour, A. Gebrewold, and B. M. Altura, "Optical spectroscopy and cerebral vascular effects of alcohol in the intact brain: effects on tissue deoxyhemoglobin, blood content, and reduced cytochrome oxidase," Alcohol. Clin. Exp. Res. 17, 1319-1324 (1993).

15. B. Chance, E. Borer, A. Evans, G. Holtom, J. Kent, M. Maris, K. Mccully, J. Northrop, and M. Shinkwin, "Optical and nuclear-magnetic-resonance studies of hypoxia in human tissue and tumors, ” Ann. NY Acad. Sci. 551, 1-16 (1988).

16. E. Gratton, S. Fantini, M. A. Franceschini, G. Gratton, and M. Fabiani, "Measurements of scattering and absorption changes in muscle and brain," Philos. T. Roy. Soc. B 352, 727-735 (1997).

17.S. S. Kety and C. F. Schmidt, "The effects of altered arterial tensions of carbon dioxide and oxygen on cerebral blood flow and cerebral oxygen consumption of normal young men,” J. Clin. Invest. 27, 484-491 (1948).

18.T. Q. Duong, C. Iadecola, and S. G. Kim, "Effect of hyperoxia, hypercapnia, and hypoxia on cerebral interstitial oxygen tension and cerebral blood flow," Magnet. Reson. Med. 45, 61-70 (2001). 